\title{
Path Durations for Use in the Stochastic-Method Simulation of Ground Motions
}

\author{
by David M. Boore and Eric M. Thompson
}

\begin{abstract}
The stochastic method of ground-motion simulation assumes that the energy in a target spectrum is spread over a duration $D_{\mathrm{T}}$. $D_{\mathrm{T}}$ is generally decomposed into the duration due to source effects $\left(D_{\mathrm{S}}\right)$ and to path effects $\left(D_{\mathrm{P}}\right)$. For the most commonly used source, seismological theory directly relates $D_{\mathrm{S}}$ to the source corner frequency, accounting for the magnitude scaling of $D_{\mathrm{T}}$. In contrast, $D_{\mathrm{P}}$ is related to propagation effects that are more difficult to represent by analytic equations based on the physics of the process. We are primarily motivated to revisit $D_{\mathrm{T}}$ because the function currently employed by many implementations of the stochastic method for active tectonic regions underpredicts observed durations, leading to an overprediction of ground motions for a given target spectrum. Further, there is some inconsistency in the literature regarding which empirical duration corresponds to $D_{\mathrm{T}}$. Thus, we begin by clarifying the relationship between empirical durations and $D_{\mathrm{T}}$ as used in the first author's implementation of the stochastic method, and then we develop a new $D_{\mathrm{P}}$ relationship. The new $D_{\mathrm{P}}$ function gives significantly longer durations than in the previous $D_{\mathrm{P}}$ function, but the relative contribution of $D_{\mathrm{P}}$ to $D_{\mathrm{T}}$ still diminishes with increasing magnitude. Thus, this correction is more important for small events or subfaults of larger events modeled with the stochastic finite-fault method.
\end{abstract}

\section{Introduction}

The stochastic method of simulating ground motions assumes that the spectral content of the ground motion at a site is given by a combination of seismological theory and empirically determined parameters, and that this energy is distributed randomly over a specified duration. As originally formulated (Hanks and McGuire, 1981; Boore, 1983), this duration was simply given as the inverse of the earthquake source corner frequency, assuming a simple single-cornerfrequency source model. This implies that the duration at all distances is the same, including at the source. Herrmann (1985) recognized that the duration is also a function of distance from the source. The duration increases with distance for a number of reasons, including separation of arrival times for wave types traveling at different speeds, scattering, reflections at layer interfaces and the free surface, and trapping of waves within sedimentary basins. Herrmann (1985) proposed that the total duration $D_{\mathrm{T}}$ should be the addition of the source duration $D_{\mathrm{S}}$ and path duration $D_{\mathrm{P}}$. Based on synthetic seismograms for a laterally uniform layered velocity model, Herrmann proposed the simple function $D_{\mathrm{P}}=0.05 R$ for the path duration, in $\mathrm{s}$, for a source-to-site distance $R$, in $\mathrm{km}$. One-dimensional synthetics such as this account for some, but not all, of the factors that increase duration with distance listed above; so the $0.05 R$ term should be considered a lower bound of the path duration. Nevertheless, this path duration was adopted by the first author for use in stochastic-method simulations in western North America and has been used for many years by a number of researchers (although more complicated functions have been used by some, such as Atkinson and Boore, 1995, in simulations in eastern North America). The recent carefully screened large database developed for the Pacific Earthquake Engineering Center (PEER) Next Generation Attenuation (NGA)-West2 project (Ancheta et al., 2014) makes this an opportune time to revisit the duration used in stochastic-method simulations in active tectonic regions.

Our goal in this article is to develop an equation for path duration that can be used in the first author's formulation of the stochastic method, as implemented in the stochasticmethod simulations suite of programs (Boore, 2005). In that formulation, $D_{\mathrm{T}}=D_{\mathrm{S}}+D_{\mathrm{P}}$. There have been several recent studies that have obtained equations for various duration definitions as functions of source, path, and site parameters. Some of these studies (Kempton and Stewart, 2006; Ghofrani and Atkinson, 2014) represent duration as an additive function of source, path, and site parameters, whereas others (Bommer et al., 2009; Bora et al., 2014; Lee and Green, 2014) have more complicated functions involving the natural $\log$ of duration as an additive function of source and site terms, with an additional term that couples source, path, or site parameters. Our initial strategy was to use those equations 
to guide a mixed-effects regression analysis using the new NGA-West2 dataset (Ancheta et al., 2014). Although all the different formulations fit the data well, we prefer the additive formulation of Kempton and Stewart (2006) because it makes sense physically because the source and path should contribute to the total duration independently (just as the stochastic method separates the effect of the source and path on the Fourier spectra into independent components). Additionally, it allows us to model only the path duration by subtracting the source duration given by a commonly used source model, which provides a means for adjusting the source duration through the corner frequency and the stress parameter. For simplicity, we choose to subjectively fit a piecewise-linear function to medians of an empirically determined duration parameter rather than adopt the formal logarithmic mixed-effects regression approach of Kempton and Stewart (2006).

To accomplish our goal, we had to develop a method of relating the duration parameters used in the stochastic method simulations to durations observed in ground motions. This is not as trivial as it might initially seem because there are many different possible definitions of groundmotion duration that could be intuitively equated to $D_{\mathrm{T}}$, and also because the duration parameter must be consistent with the parameters that define the envelope function of the stochastic method (equation 24 in Boore, 2003).

We first describe the duration parameter that we have obtained from the time series used in developing the NGA-West2 database and then discuss the development of the new function for $D_{\mathrm{P}}$. This is followed by a residual analysis of the new function and a brief comparison with previous equations for ground-motion duration. We then compare the magnitude dependence of several ground-motion intensity measures (GMIMs) from data and from simulations made using the original and revised functions for $D_{\mathrm{P}}$.

\section{The Measure of Duration Used in this Study}

A number of ground-motion duration measures have been proposed. Bommer and Martínez-Pereira (1999) found that the measures could be classified as one of three types. The type of concern to us is significant duration (introduced by Trifunac and Brady, 1975), for which the duration of a record is based on the integral of the squared acceleration time series, although others have generalized the definition to allow for the use of the squared velocity time series. We use significant durations based on the acceleration series because of its close association with the root mean square (rms) acceleration, which is at the core of the stochastic method. For any one record, a number of significant duration measures can be computed. For example, durations can be defined between any two fractions of the cumulative integral of the squared acceleration (Husid plots), normalized to unity at the end of the record. Two common measures are the intervals between the time at which the integral reaches $5 \%$ and $75 \%$ of the maximum and $5 \%$ and $95 \%$ of the maximum (e.g., Ou and Herrmann, 1990a,b; Kempton and Stewart, 2006; Bommer et al., 2009). Different terminologies have been used for these measures, such as $D_{a 5-95}$ (Kempton and Stewart, 2006, in which $a$ indicates the use of acceleration rather than velocity) and $D_{\mathrm{SR} 5 \%-95 \%}$ (Bommer et al., 2009); for brevity we use $D_{95}$ (and similarly for other fractions) because our measure always starts at $5 \%$ of the maximum and is always calculated from the acceleration time series. Other measures are determined by the duration required for a GMIM determined from the record to be equal to the value predicted from random vibration theory, given the rms of the record. The GMIMs used in these determinations of duration include the peak ground acceleration (PGA; Vanmarcke and Lai, 1980), peak ground velocity (Atkinson, 1993, 1995), and spectral acceleration at a number of periods (Bora et al., 2014). These last measures would seem to be the most relevant for the stochastic method, but the method as formulated by the first author (Boore, 1983) used $D_{95}$ to define the total duration of shaking $\left(D_{\mathrm{T}}\right)$ and then a separate duration to compute peak values, which is a function of the oscillator period. We continue to prefer this approach because the duration of groundmotion excitation is conceptually distinct from the duration of the oscillator's response (Boore and Thompson, 2012). We continue to use $D_{95}$ as the measure of duration for several reasons: continuity with the past, retaining the amplitude envelope shaping window, the availability of $D_{95}$ for the NGA-West2 database, and the ambiguity as to which GMIM to use.

The duration $D_{95}$ enters into the stochastic method in using an exponential shaping window to approximate the shape of a real seismogram. Boore (1983) was guided by the work of Saragoni and Hart (1974) in determining the shape of an exponential envelope, and he chose the envelope parameters such that the $D_{95}$ duration (computed from the simulated ground motions) was on average equal to the total duration of ground excitation $D_{\mathrm{T}}$ (the parameter used in the SMSIM programs). Figure 1 shows simulated accelerations from the stochastic-method simulation program, for two magnitudes and a distance of $100 \mathrm{~km}$. The source, path, and total durations used in the simulations, as well as $D_{95}$ calculated from the time series are given in the legend. The measured $D_{95}$ does not exactly equal $D_{\mathrm{T}}$, and there is no reason that $D_{\mathrm{T}}$ should equal $D_{95}$ for any one realization. As will be shown in a later figure, that condition is closely met on average. As it happens, the discrepancy between $D_{95}$ and $D_{\mathrm{T}}$ for the $\mathbf{M} 4, R=100 \mathrm{~km}$ record shown in Figure 1 is close to the maximum difference for the 100 simulations.

The durations we use in developing the new $D_{\mathrm{P}}$ function were computed as part of the PEER NGA-West2 project (see Data and Resources). In our analysis, we applied the same selection criteria as in Boore et al. (2014). We found that the $D_{95}$ values from the data had some undesirable characteristics that were inconsistent with the stochastic method's assumption that the simulated motions are primarily for $S$-body waves: the time at which the 0.05 fraction is reached can be too early because of strong $P$-wave energy; and the time for the 0.95 fraction to be reached can be too late, either 

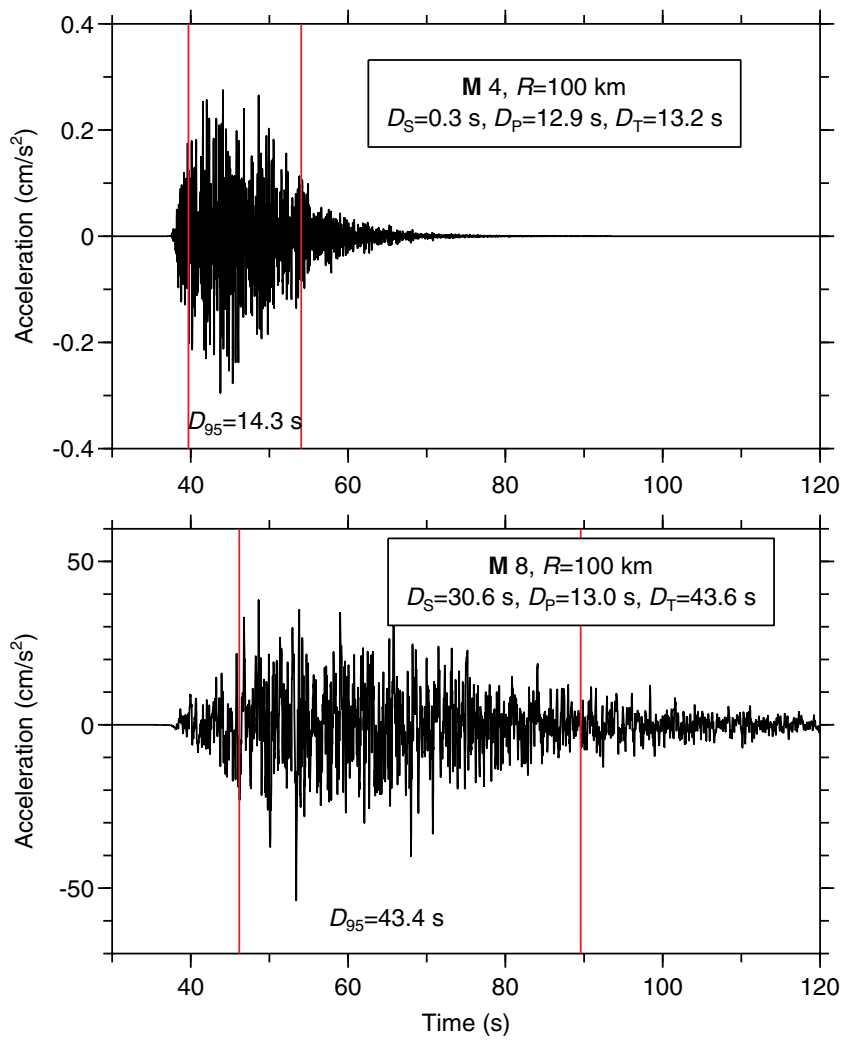

(a)
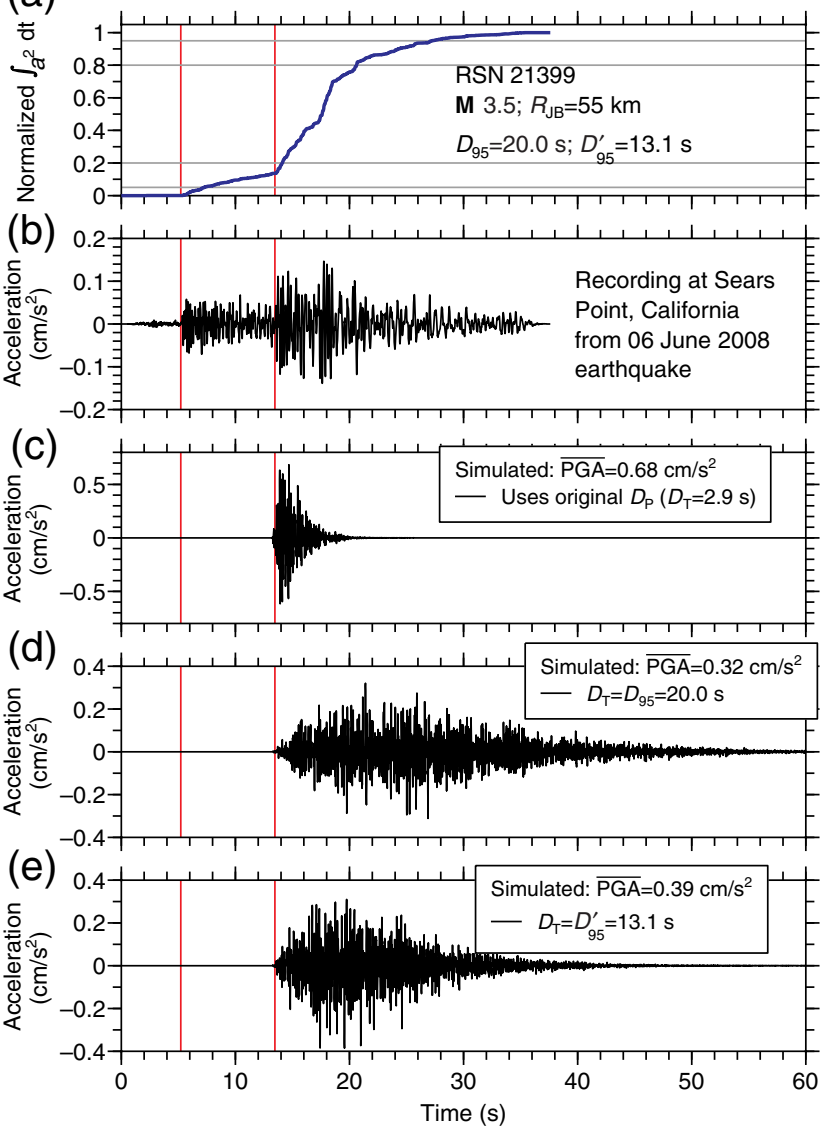

Figure 1. Acceleration time series from stochastic-method simulations, using the new path duration $D_{\mathrm{P}}$ derived in this article. The vertical lines indicate the times at which the cumulative integral of acceleration squared (a Husid plot) reaches 5\% and 95\% of the maximum value; the duration $D_{95-5}$ is the difference in these times. For brevity, we use $D_{95}$ rather than $D_{95-5}$ in this article, because the start time for defining the significant duration is always the $5 \%$ time on the normalized Husid plot. $D_{\mathrm{S}}$ is the source duration, given as $0.5 / f_{a}$, in which $f_{a}$ is the corner frequency given by equation (6) in Atkinson and Silva (2000). The $D_{\mathrm{P}}$ for the records from the two magnitude events differ slightly because the distances used in $D_{\mathrm{P}}$ function have been adjusted for the finite extent of the faults; the distance for the $\mathbf{M} 8$ event is slightly larger than that for the $\mathbf{M} 4$ event. The color version of this figure is available only in the electronic edition.

because of late arriving longer period energy (presumably surface waves) or the presence of strong aftershocks. The problem of an early time for the 0.05 fraction is illustrated in Figure 2. The Husid plot of the recording is given in the top panel, and the record (second to the top panel) is compared with stochastic simulations in which all parameters are held constant except for $D_{\mathrm{T}}$. The arrival time of the $P$ and $S$ waves are labeled on each plot, the simulations are aligned to coincide with the $S$-wave arrival. $D_{\mathrm{T}}$ and the median PGA from 800 simulations $(\overline{\mathrm{PGA}})$ are given for each simulation.

The main purpose of Figure 2 is to demonstrate that in some cases the $5 \%$ level for data is reached substantially earlier than the $S$-wave arrival. Parts c, d, and e of the figure, however, also demonstrate the consequences of the different durations for simulated ground motions. Figure 2c shows that the original duration used in stochastic-method simula-

Figure 2. The Husid plot (graph a) from the acceleration record shown in graph $b$. For this record the 0.05 level is reached near the $P$-wave arrival. Graphs (c), (d), and (e) show simulations using $D_{\mathrm{T}}$ values equal to the original path duration and the $D_{95}$ and $D_{95}^{\prime}$ durations obtained for the recording $\left(D_{95}^{\prime}\right.$ is the effective duration used in this study), respectively. The change of the ordinate scale of part (c) compared to the other two simulations. The time series shown for each duration model is the one simulation out of 800 that had a PGA closest to $\overline{\mathrm{PGA}}$. The vertical lines indicate the $P$ - and $S$-wave arrivals (the simulations have been aligned on the $S$-wave arrival). The color version of this figure is available only in the electronic edition.

tion is too short, whereas Figure 2d shows that using $D_{95}$ from the recorded time series overestimates the path duration because of the presence of strong $P$-wave energy. Figure $2 \mathrm{e}$ shows the duration parameter that we propose in this paper (defined below as $D_{95}^{\prime}$ ). The simulations can be compared in terms of the total duration, but also in terms of their respective $\overline{\text { PGA: }}$ the larger the $D_{\mathrm{T}}$, the smaller the amplitude (by approximately the inverse of the square root of $D_{\mathrm{T}}$ ) because the energy is spread across a larger time window. The median PGA (indicated by $\overline{\mathrm{PGA}}$ ) for the 800 simulations is given in the legends; as expected, the ratios of any two simulations is close to the square root of the ratio of their durations. There has been no attempt to adjust the simulation parameters to match the observed PGA, as the relative durations were of most interest in this article. The Fourier acceleration spectrum used in each simulation is not that of the observed record but is 

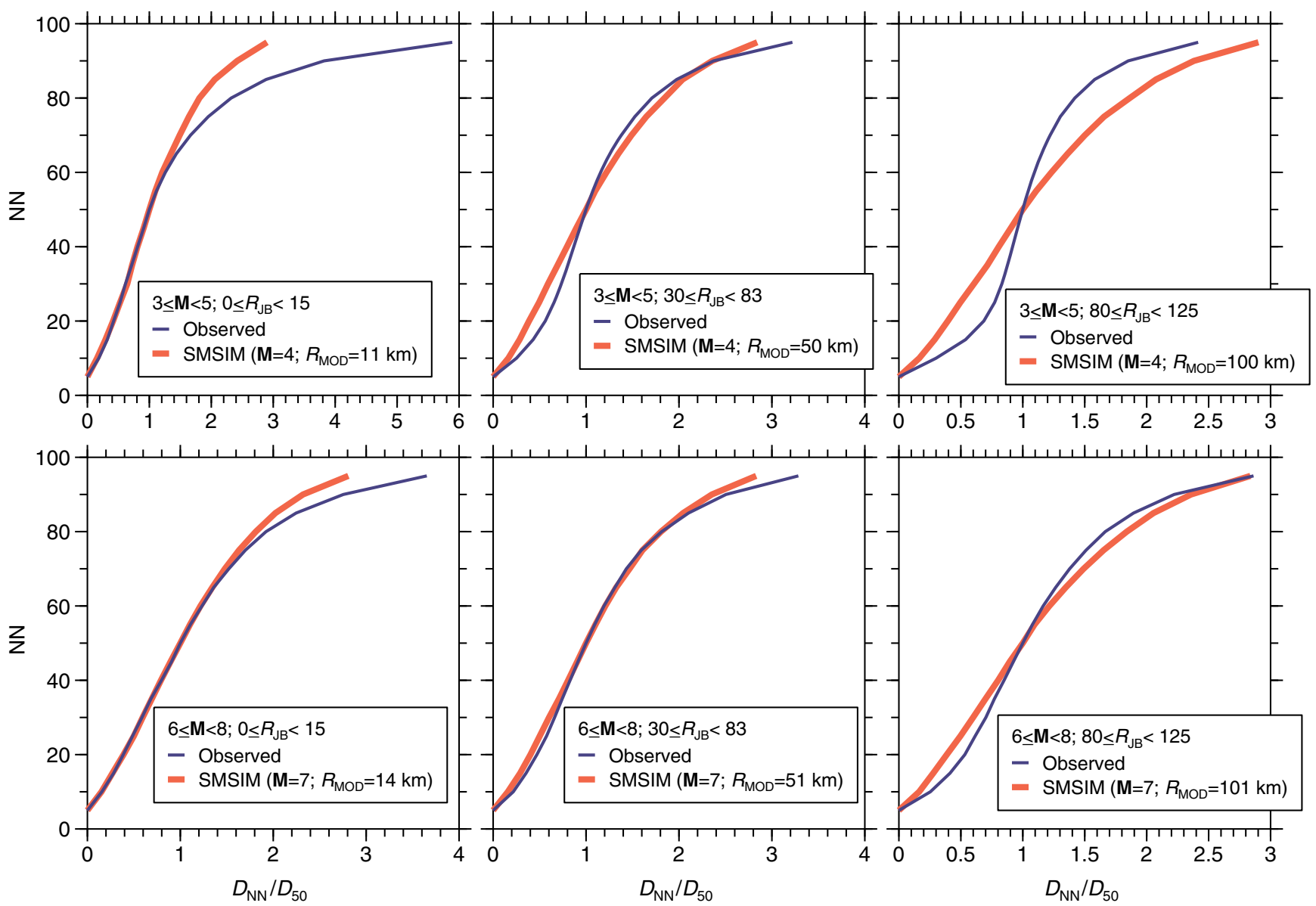

Figure 3. Comparison of Husid plots (in percent) using normalized significant durations for the abscissa. The curves are based on medians of the Husid curves for NGA-West 2 records in the $\mathbf{M}$ and $R_{\mathrm{JB}}$ bins indicated for the observations and for 100 simulations for $\mathbf{M}$ and $R_{\mathrm{MOD}}$ pairs $(4,11),(4,50),(4,100),(7,14),(7,51)$, and $(7,101)$ for stochastic-method simulation, in which $R_{\mathrm{MOD}}$ is the distance used in the stochastic-method simulation point-source simulations. It is the source-site distance, modified to account for finite-fault effects using the formulation of Atkinson and Silva (2000). The color version of this figure is available only in the electronic edition.

from the particular model used for the stochastic method (all parameters but the duration were the same for the simulations).

As discussed earlier, in the stochastic method we use the $D_{95}$ measure of duration. Figure 2 shows that strong $P$-wave arrivals can lead to an elongation of the duration due to an early time at which the 5\% level is reached. This early start to the duration measure is incompatible with the stochastic-method assumption that simulations are for the $S$-wave contribution to the ground motion. Similar problems can occur due to a delay of the time at which the $95 \%$ level is reached. These effects imply that the shape of the envelope of ground motion from data may be inconsistent with that assumed in the simulations.

To investigate this in detail, we compared the Husid plots from the NGA-West2 database to those from simulations. To remove any magnitude and distance dependencies of the Husid plots, we plotted the Husid percentage NN against the ratios of the duration $D_{\mathrm{NN}}$ to $D_{50}$. In Figure 3, we show such plots both for simulated motions at six representative pairs of magnitude and distance (15 pairs were used in the analysis) and for observations in magnitude and distance bins centered on the pairs used in the simulations.
The observations are based on the large NGA-West 2 database, in which the number of records ranges from 332 for the M 6-8 and $R_{\mathrm{JB}}=0-15 \mathrm{~km}$ graph to 3752 for the M 3-5 and $R_{\mathrm{JB}}=30-83 \mathrm{~km}$ graph $\left(R_{\mathrm{JB}}\right.$ is the shortest horizontal distance from a site to the vertical projection of the rupture surface to the Earth's surface). The results in Figure 3 imply that there is a mismatch between the assumed shapes of the time-domain envelopes for smaller events (compare the results from observations and simulations), with relatively good agreement for larger magnitudes. To avoid the instability due to the early and late times at which the 5\% and 95\% levels are reached, respectively, we propose an effective duration $D_{95}^{\prime}$ :

$$
D_{95}^{\prime}=2.0\left(D_{80}-D_{20}\right) \text {. }
$$

The scale factor of 2.0 is based on the average of the ratio of $D_{95}$ to $D_{80}-D_{20}$ from the simulations. The choice of $D_{20}$ and $D_{80}$ was subjective, based on plots such as those in Figure 3, the intent being to capture the duration due to the main $S$-wave motion. Equation (1) was used for the 15,923 records in the NGA-West2 database that met the selection 

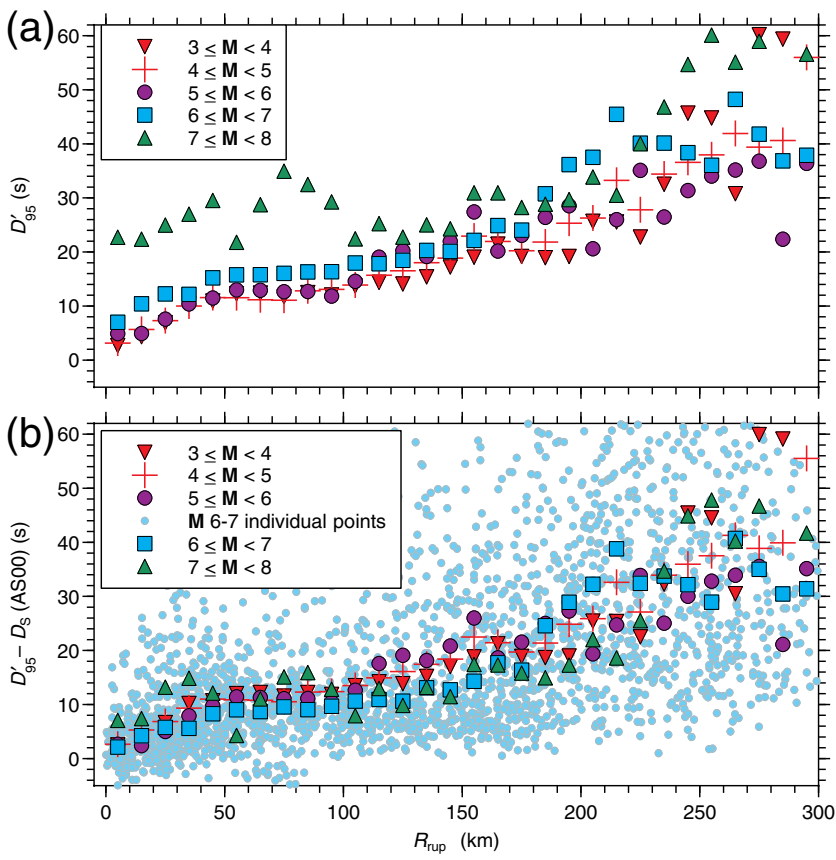

Figure 4. (a) Median values in magnitude and distance bins of the effective $D_{95}$ durations $\left(D_{95}^{\prime}\right)$ derived from the geometrical means of the durations from two horizontal components from 15,923 records from the NGA-West 2 database. (b) The medians of the effective $D_{95}$ durations after subtracting the source duration given by $0.5 / f_{a}$, in which $f_{a}$ is the corner frequency given by equation (6) in Atkinson and Silva (2000; hereafter AS00). Also shown are the individual points for the M 6-7 bin. The color version of this figure is available only in the electronic edition.

criteria of Boore et al. (2014). Median values of $D_{95}^{\prime}$ for various magnitude and distance bins are shown in Figure 4a, as a function of distance.

\section{A Revised Model for Path Durations $\left(D_{\mathrm{P}}\right)$}

As can be seen in Figure 4a, the $D_{95}^{\prime}$ durations clearly depend on distance, but they are also much larger for the biggest events than for the smaller events, illustrating that $D_{95}^{\prime}$ contains source durations $D_{\mathrm{S}}$ in addition to path durations $D_{\mathrm{P}}$. We assume that $D_{95}^{\prime}=D_{\mathrm{S}}+D_{\mathrm{P}}$ and thus isolate $D_{\mathrm{P}}$ by subtracting $D_{\mathrm{S}}$ from $D_{95}^{\prime}$. It would be ideal if we knew $D_{\mathrm{S}}$ for each event, but those are not available, and for that reason we use an estimate of $D_{\mathrm{S}}$ based on Atkinson and Silva (2000; hereafter AS00):

$$
D_{\mathrm{S}}=0.5 / f_{a},
$$

in which the source corner frequency $f_{a}$ is given by equation (6) in AS00. $f_{a}$ is the lower of two corner frequencies in their double-corner-frequency source model. (As a side note, the high-frequency spectral level of the AS00 spectral model equals that for a single-corner frequency model with a stress parameter of 88 bars, using the various model parameters in AS00.) The resulting estimate of $D_{\mathrm{P}}$ is shown in Figure 4b. To give an idea of the variability of $D_{\mathrm{P}}$, we show the individual points for the $\mathbf{M}$ 6-7 bin in addition to the median values. The variability increases with distance. For distances less than $100 \mathrm{~km} 50 \%$ of the values for each distance bin are generally within $5 \mathrm{~s}$ of the median, and the variability increases such that at a distance of $250 \mathrm{~km} 50 \%$ of the values are within about $20 \mathrm{~s}$ of the median. Subtracting $D_{\mathrm{S}}$ from $D_{95}^{\prime}$ has brought the durations from the various magnitude bins into reasonable agreement with one another, especially within $200 \mathrm{~km}$, and it is those adjusted values that we use to develop our new path duration function $D_{\mathrm{P}}$. We chose the new duration function subjectively, guided by the medians in the magnitude 6-7 range. We focus on this range for several reasons: there are a relatively larger number of observations for this range (unlike for the magnitudes 5-6 and $7-8$ ), and the observations are less likely to be influenced by the effects that caused us to introduce the effective measure of duration $D_{95}^{\prime}$ (see, Fig. 3).

The use of a subjective fit rather than one from a formal regression analysis is in keeping with the determination of a number of the parameters used in the stochastic method, such as the frequency dependence of both the quality factor and the crustal amplification. We could have obtained similar results by a regression analysis that used the proper weighting of the observed durations, but we felt that it was more efficient to use our judgment in determining $D_{\mathrm{P}}$. Our subjective fit is shown by the thick line in Figure 5. The coefficients of the new duration function are given in Table 1. Figure 5 also shows the original $D_{\mathrm{P}}(0.05 R)$, as well as the path durations developed by Atkinson and Boore (1995), Atkinson (1995), and G. Atkinson (personal comm., 2014) for crustal earthquakes in eastern North America, Cascadia, and active tectonic regions, respectively. The new durations are consistently larger than the previous ones for eastern North America and Cascadia but are quite similar to the one proposed by Atkinson for active tectonic regions, at least for distances within about $180 \mathrm{~km}$. Atkinson's 2014 duration was developed from consideration of the original $D_{\mathrm{P}}$ as well as the frequency-dependent durations of Raoof et al. (1999). Both our new duration and Atkinson's 2014 duration show a decrease with distance starting near $50 \mathrm{~km}$; this could be a result of critical angle reflections, which can also lead to a change in geometrical spreading at about this distance (e.g., Raoof et al., 1999). The larger slope of our revised duration at distances beyond about $150 \mathrm{~km}$ might be a result of the increasing importance of surface waves and scattered energy. We show the consequences of our revised duration function in a later section.

\section{Comparisons with Other Duration Functions}

The new duration function is compared with the duration functions of Kempton and Stewart (2006; hereafter KS06), Bommer et al. (2009; hereafter Bea09), and Bora et al. (2014; hereafter Bea14) in Figure 6. Because the durations given by those authors are total durations, we added back $D_{\mathrm{S}}$ from AS00 to our new $D_{\mathrm{P}}$. In evaluating the KS06, Bea09, and Bea14 duration functions, we had to make choices for two parameters not included in our function: the 


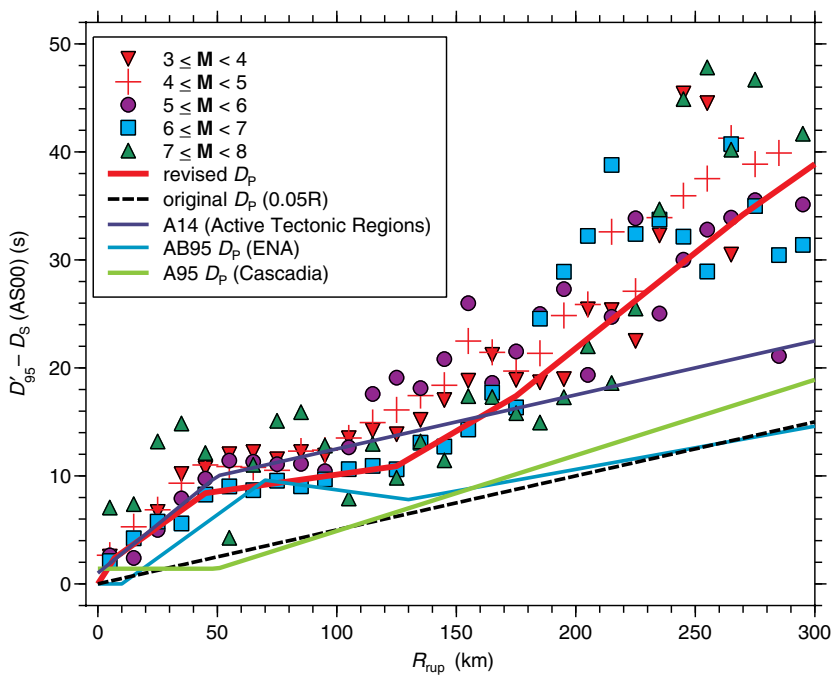

Figure 5. The medians, in various magnitude and distance bins, of $D_{\mathrm{P}}=D_{95}^{\prime}-D_{\mathrm{S}}$, in which the source duration $D_{\mathrm{S}}$ is given by $0.5 / f_{a}$ (we use equation 6 in AS00 for the corner frequency $f_{a}$ ). Guided primarily by the medians for the $\mathbf{M}$ 6-7 range, particularly for $R_{\text {rup }}$ less than about $200 \mathrm{~km}$, we subjectively derived a path duration function consisting of joined linear segments. For comparison, the original $D_{\mathrm{P}}$ is shown, as well as the $D_{\mathrm{P}}$ functions used in stochastic-method simulations for active tectonic regions by G. Atkinson (personal comm., 2014; A14), the Cascadia region of western North America by Atkinson (1995; A95), and eastern North America (ENA) by Atkinson and Boore (1995; AB95). The color version of this figure is available only in the electronic edition.

average shear-wave velocity in the upper $30 \mathrm{~m}\left(V_{S 30}\right)$ and the depth to the top of rupture $\left(Z_{\mathrm{TOR}}\right)$ for Bea09. For $V_{S 30}$, we show results for soil-like and rocklike values $(310 \mathrm{~m} / \mathrm{s}$ and $620 \mathrm{~m} / \mathrm{s}$, respectively). $Z_{\mathrm{TOR}}$ is a function of magnitude, and we used representative values, shown in the graph legends, based on histograms from the dataset used in this article. The Bea14 duration is a function of $R_{\mathrm{JB}}$, whereas the rest are functions of $R_{\text {rup }}$ (the shortest distance from the site to the rupture surface). We converted from $R_{\mathrm{JB}}$ to $R_{\text {rup }}$ using magnitude-dependent effective depths obtained by the first author for strike-slip earthquakes; these depths go from $8 \mathrm{~km}$ for $\mathbf{M} 3.75$ to $0 \mathrm{~km}$ for $\mathbf{M}$ 7.5. Considering all but the $\mathbf{M} 7.5$ graph, we make these observations: (1) the total duration computed using the original $D_{\mathrm{P}}$ is less than that from the other studies; (2) in contrast, the total duration using the revised $D_{\mathrm{P}}$ is in reasonable agreement with the others for distances within about $50 \mathrm{~km}$; (3) the total duration using the revised $D_{\mathrm{P}}$ is generally lower than the other values for greater distances. For M 7.5 there is more difference between the durations, and it is harder to make general observations.

\section{Residual Analysis}

Our new duration function is simpler than those discussed in the previous section, as the only predictor variables are $\mathbf{M}$ and $R_{\text {rup }}$. The KS06 base model assumes a similar independence of the magnitude and distance scaling but also includes scaling for other factors such as $V_{S 30}$; Bea09 include terms
Table 1

The New Path Duration Model

\begin{tabular}{cc}
\hline$R_{\text {rup }}(\mathrm{km})$ & $D_{\mathrm{P}}(\mathrm{s})$ \\
\hline 0 & 0 \\
7 & 2.4 \\
45 & 8.4 \\
125 & 10.9 \\
175 & 17.4 \\
270 & 34.2 \\
Slope of last segment & 0.156 \\
\hline
\end{tabular}

Values for nontabulated distances are given by linear interpolation of the tabulated values (in terms of duration and distance, not logarithms of these quantities). Durations for distance beyond the last tabulated distance are given by $D_{\mathrm{P}}(R)=D_{\mathrm{P}}\left(R_{\text {last }}\right)+$ slope $\times\left(R-R_{\text {last }}\right)$.

for $V_{S 30}$ and $Z_{\mathrm{TOR}}$, as well as an interaction term between distance and magnitude. It is therefore of interest to analyze the residuals of our duration function for missing trends with respect to the predictor variables, including those employed by other duration functions. To compare with the other functions, we add back the source duration to our new path duration function as we did in the previous section and perform a mixed-effects regression, with each earthquake being treated as a random effect (i.e., we assume a correlation between the durations for each earthquake). We used the same dataset of 15,923 data points as used in developing the new duration function. The inter- and intraevent residuals are shown in Figure 7 for various predictor variables. The dependencies are small, indicating that our new duration function works remarkably well, given its simplicity. There are small trends, however, with respect to $\mathbf{M}, Z_{\mathrm{TOR}}$, and $V_{S 30}$. We could derive adjustment factors to account for these trends, but we chose not to do so for several reasons. Some dependence on $\mathbf{M}$ is expected because we weighted the M 6-7 values more heavily than the durations from the other magnitude bins in deriving our new duration function; residual plots for a path duration guided by the median of the M-bin medians, which gives more weight to the medians for smaller magnitude, showed almost no dependence on $\mathbf{M}$. The negative correlation with $Z_{\mathrm{TOR}}$ (implying that the observed total durations are somewhat shorter than our predicted total durations) might not be the result of a $Z_{\mathrm{TOR}}$ dependence of the path duration but rather the result of the source duration being shorter for the buried ruptures, as would happen if the stress parameter for those events was higher than the average stress parameter implied by the AS00 model (such an increase has been proposed by some authors, such as Pitarka et al., 2009). Finally, the $V_{S 30}$ dependence is small in the range of $V_{S 30}$ of most concern for applications of stochastic-method simulation (rocklike conditions; these can be used to provide input motions for the computation of local site response for sites with low $V_{S 30}$ values). We think any advantages of including adjustments for the small trends associated with $\mathbf{M}, Z_{\mathrm{TOR}}$, and $V_{S 30}$ do not outweigh the simplicity of our duration model in applications. 

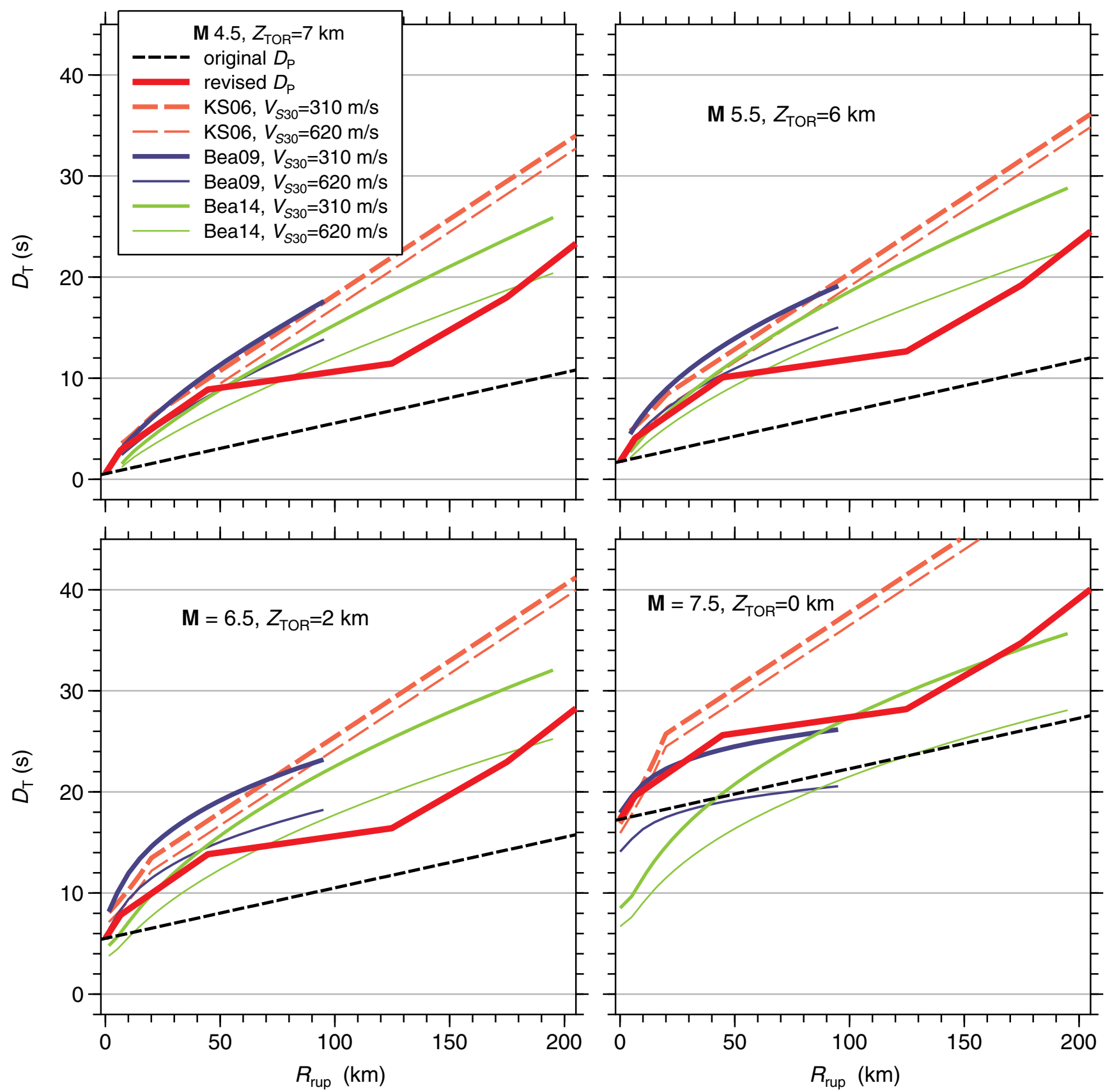

Figure 6. Comparison of ground-motion durations $\left(D_{\mathrm{T}}\right)$. Kempton and Stewart (2006; hereafter KS06); using their equations (13) (base model) and (19) (near-fault adjustment); Bommer et al. (2009; hereafter Bea09); Bora et al. (2014; hereafter Bea14). The source duration for the AS00 model has been added to the original and revised $D_{\mathrm{P}}$ in the graphs. The distances limits for each function are honored in making the plot. The color version of this figure is available only in the electronic edition.

\section{Implications of the Revised Model for Predicted Ground Motions}

Comparisons of Simulations Using the Original and Revised Path Duration Functions

As stated earlier, the envelope parameters for use in time-domain simulations in the stochastic-method simulation programs were chosen so that $D_{95}=D_{\mathrm{S}}+D_{\mathrm{P}}$ on average, for a representative source function. The envelope parameter given in Boore (1983) was based on a relatively few number of simulations. We revisited this parameter by doing 100 simulations for 15 magnitude and distance pairs. We found that we had to change the envelope parameter $\left(f_{-} t b 2 t e\right.$ in the stochastic-method simulation programs) from 2.0 to 2.12 . With this slight revision, we obtained the correspondence between $D_{95}$ and $D_{\mathrm{T}}$ shown in Figure 8 .

Using the new envelope parameter, the 5\% damped relative displacement response spectra (SD) from simulated 

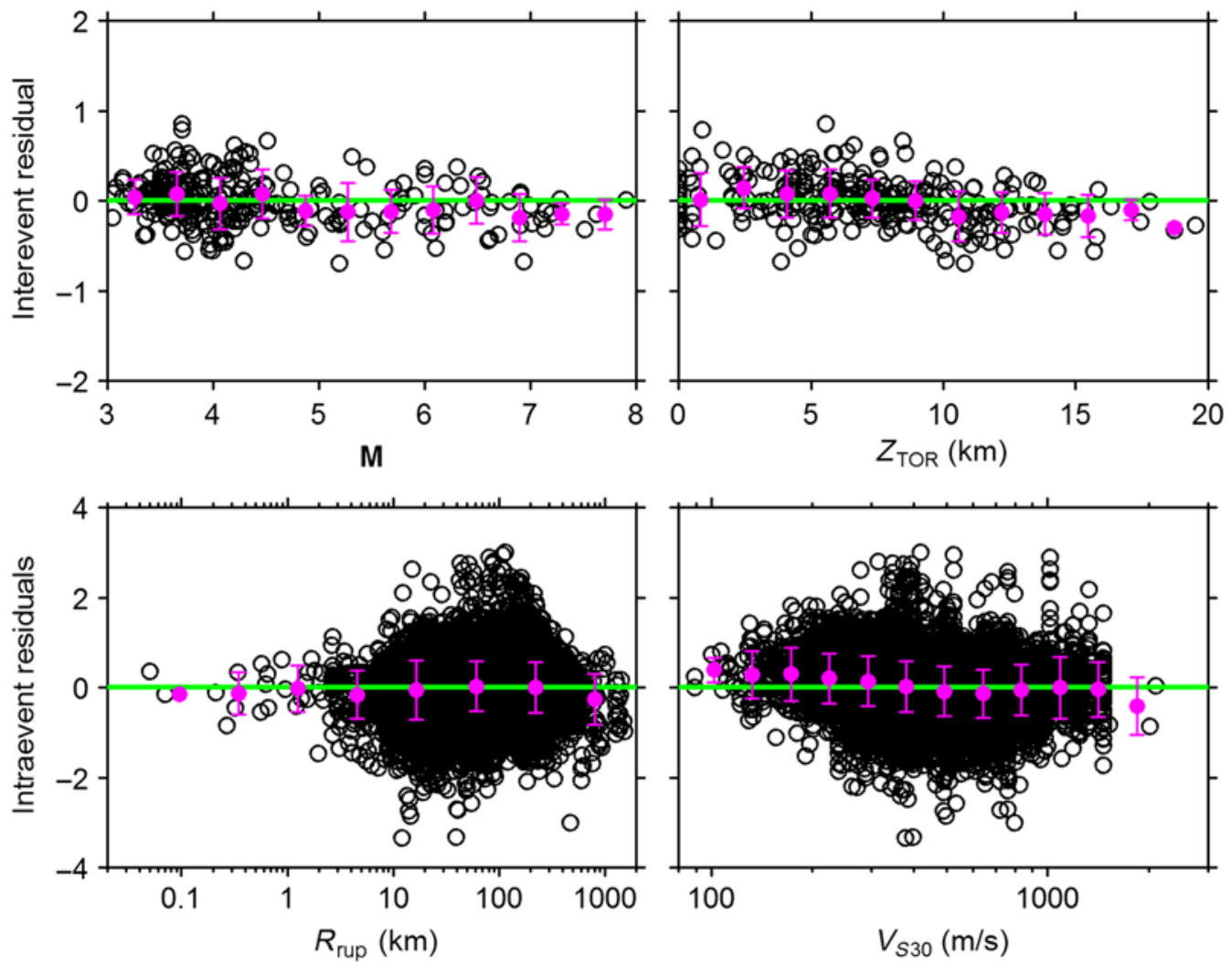

Figure 7. Residuals from a mixed-effects analysis. The residuals are defined as $\ln \left(D_{95}^{\prime}\right)-\ln \left(D_{\mathrm{S}}+D_{\mathrm{P}}\right)$, in which the first term is from observations, and the second term uses the sum of the revised $D_{\mathrm{P}}$ and the AS00 source duration. Shown are the individual points (open circles) and the means (solid circles) and \pm 1 standard deviation of the data about the means within bins of the predictor variables; the standard error of the means are so small that they would generally be difficult to see in the graphs. The color version of this figure is available only in the electronic edition.

motions using the original and revised path durations are compared in Figure 9. The simulations were done for two magnitudes (4.0 and 7.0) at a distance of $60 \mathrm{~km}$ (this distance is a compromise between the close and far distances at which the source or the path durations dominate the results, respectively, and yet the distance is one for which the ground motions might be of engineering significance). As expected, the revised durations lead to smaller motions, because the same spectral energy is being spread out over a longer duration. The ratio of the motions will be less than the square root of the inverse ratio of their durations, because the rms-to-peak factor increases with duration, and this tends to counteract the reduction in rms with increasing duration. For a fixed distance the contribution of the source duration to the total duration increases with magnitude, and therefore the differences in the response spectral amplitudes from simulations using the original and the revised path durations are smaller for the M 7 event than for the M 4 event.

\section{M scaling}

We now compare the magnitude scaling of the 5\% damped pseudospectral acceleration (PSA) of simulations with data. Figure 10 is an updated version of a figure in
Boore (2014), showing oscillator period $T_{\text {OSC }}=0.2 \mathrm{~s}$ PSA using the revised path duration function (as well as the original path duration function). We chose $T_{\mathrm{OSC}}=0.2 \mathrm{~s}$ because it is one of the main periods used in design maps in some recent building codes (see Data and Resources for some references) and because the difference between the original and revised durations increases with decreasing period (as shown in Fig. 9). Similar plots of magnitude scaling for periods of $1.0 \mathrm{~s}$ and $4.0 \mathrm{~s}$ are in Boore (2014) (but using the original duration function). The observations are for strike-slip events, and the observed motions have been adjusted to $V_{S 30}$ of $620 \mathrm{~m} / \mathrm{s}$, using the Boore et al. (2014; hereafter BSSA14) equations $\left(620 \mathrm{~m} / \mathrm{s}\right.$ is the value of $V_{S 30}$ for the velocity model used to derive the crustal amplifications used in the simulations). The predictions from the BSSA14 ground-motion prediction equations (GMPEs) are shown in addition to the individual data points. Except for $D_{\mathrm{P}}$ and $f_{-} t b 2 t e$, all simulation parameters are those used by AS00; there has been no attempt to adjust the parameters to obtain a better fit to the data. The apparently small differences in the simulations are consistent with the differences shown in Figure 9. Overall, the simulations are in moderateto-good agreement with the data. There is a relative lack of data for $\mathbf{M}$ between about 5.5 and 6.4; as a result, the 


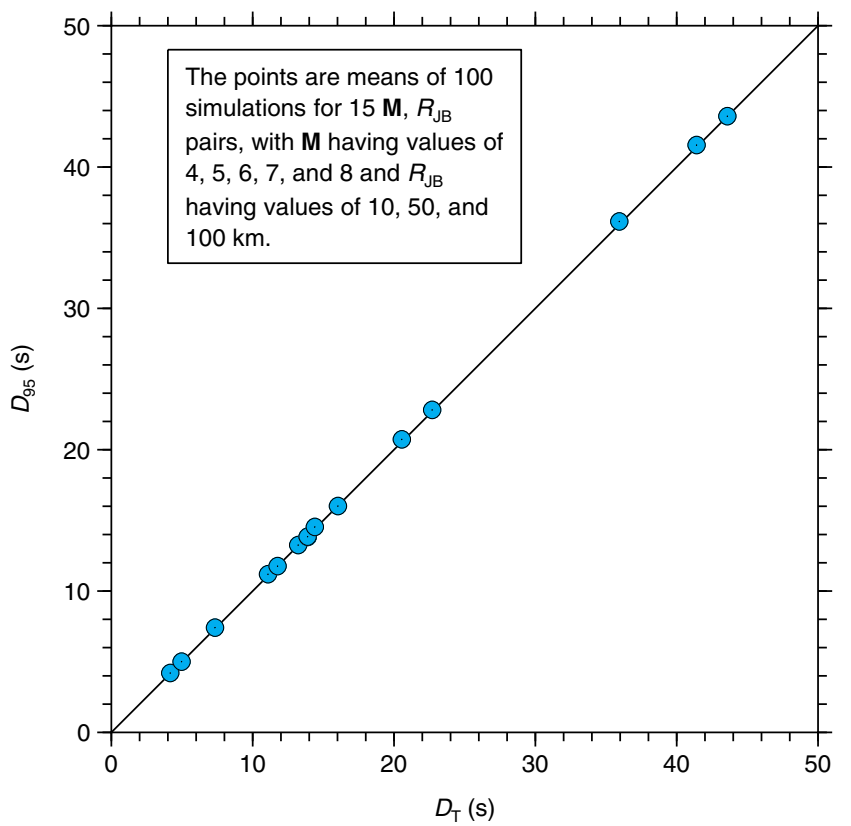

Figure 8. A comparison of the $D_{95}$ values from simulations at 15 pairs of magnitude and distance values (as given in the legend) and the duration of ground motion $\left(D_{\mathrm{T}}\right)$ for each pair of magnitude and distance. The new value for the exponential envelope adjustment factor used in the stochastic-method simulation programs $\left(f_{\_} t b 2 t e=2.12\right)$ was used in the simulations. This is an updated version of figure 2 in Boore (1983). The color version of this figure is available only in the electronic edition.

BSSA14 GMPEs are not well constrained in this region, which marks the transition from a steeper to a shallower M scaling. The revised duration function gives a better fit to the small magnitude observations at larger distances than does the original duration function. Boore (2014) presented analogous figures for longer periods, but for those cases there is no appreciable difference between the PSA using the original and revised durations.

\section{Revisions of Published Results}

The new duration function may require modification of results in two of our previous papers. Making these modifications now is beyond the scope of this paper, but we mention them here for completeness. In Boore (2009), it was assumed by mistake that the duration used in the stochastic-method simulations was $D_{75}$ rather than $D_{95}$; correcting this mistake will modify the discussion centered on figures 9 and 10 in Boore (2009).

The second study that may require modification is by Boore and Thompson (2012; hereafter BT12), in which adjustments for the durations used to obtain peak motion estimates from random-vibration theory were obtained by comparing time-domain and random-vibration calculations. The results of the time-domain calculations used to obtain the western North America adjustment factors will be different because of the new duration function and exponential envelope factor $f_{-} t 2 b e$. Preliminary calculations, not shown here, suggest that the modifications to BT12 will be minor.

\section{Discussion and Conclusions}

The most commonly used existing formulation of the stochastic method (e.g., Boore, 2003, 2005) assumes that the duration of ground motion is given by a frequencyindependent sum of a source duration and a path duration, despite the observations of Herrmann and colleagues that the duration is frequency dependent (e.g., Raoof et al., 1999; Malagnini et al., 2007). In defense of the stochastic method, it has been used for many years, and comparisons with data
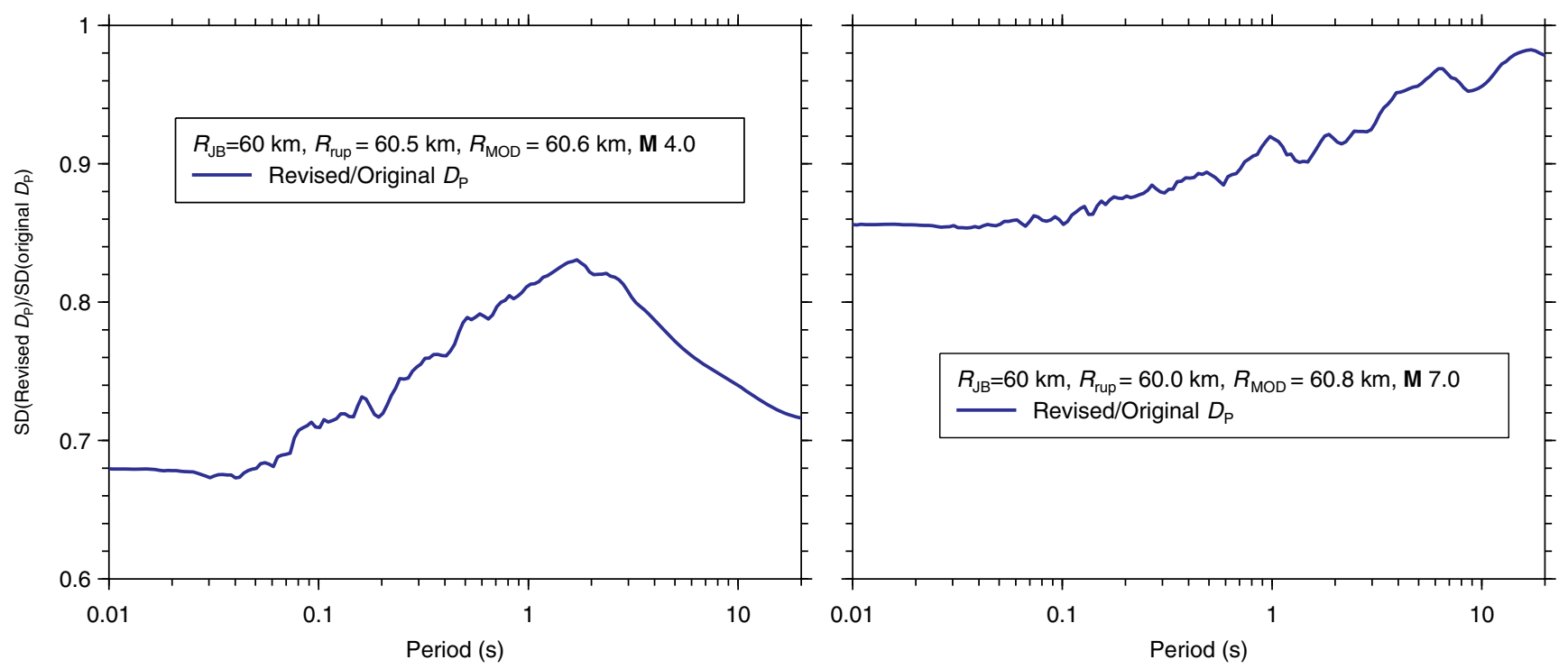

Figure 9. Ratios of the 5\% damped relative displacement (SD) response spectra from time-domain stochastic-method simulations at a distance of $60 \mathrm{~km}$ for magnitude 4 and 7 earthquakes using the revised and the original path durations. The color version of this figure is available only in the electronic edition. 
simulations use the AS00 source, $\boldsymbol{\kappa}_{0}=0.03 \mathrm{~s}$, and unless noted, the AS00 finite-fault adjustment factor and a conversion from $R_{\mathrm{JB}}$ to $R_{\mathrm{rup}}$ derived by $D$. Boore
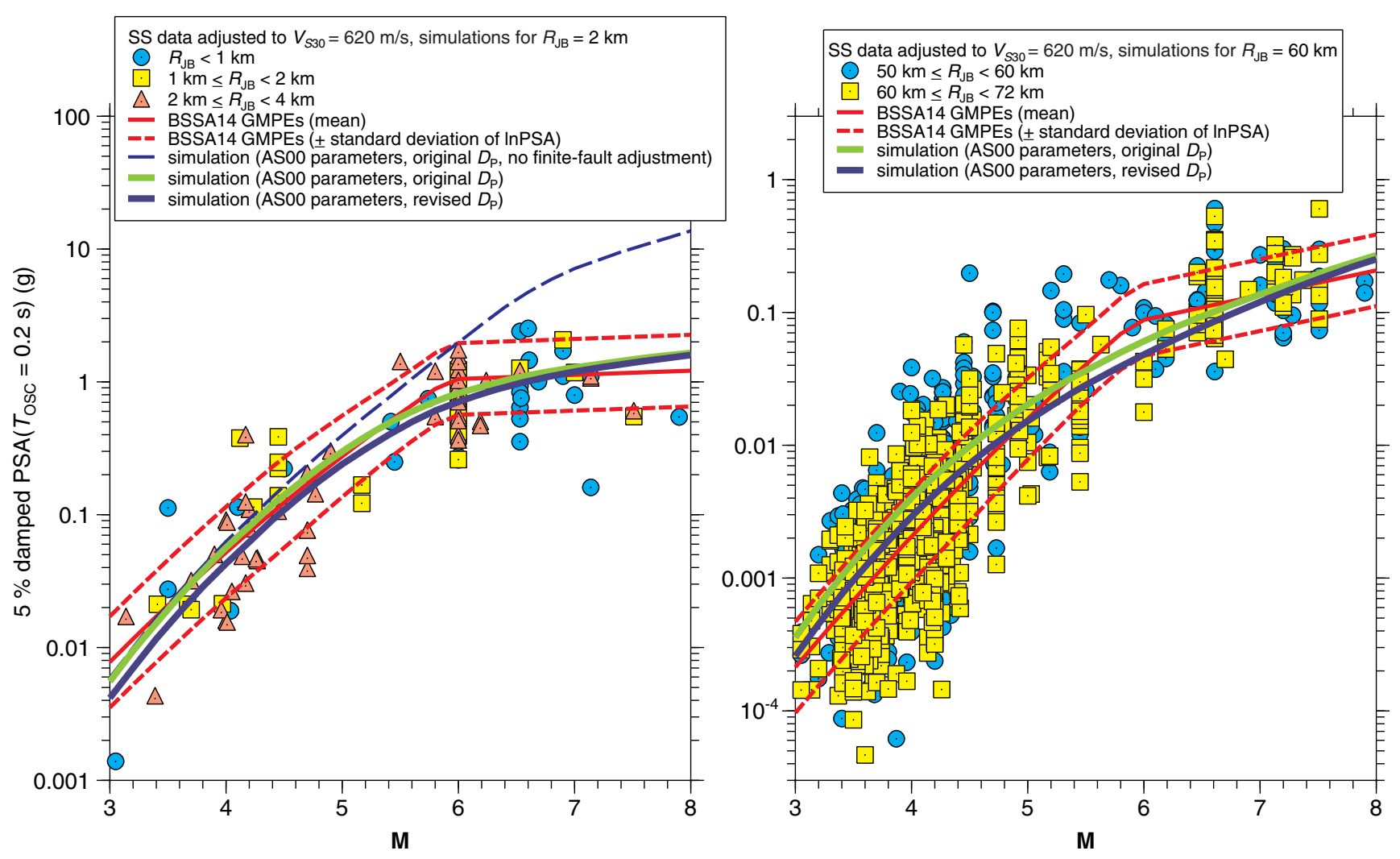

Figure 10. Magnitude scaling for $T_{\mathrm{OSC}}=0.2 \mathrm{~s}$ PSA near-fault (left graph) and intermediate-fault (right graph) distances, for strike-slip (SS) earthquakes. The symbols are data from the NGA-West 2 database, adjusted to $V_{S 30}=620 \mathrm{~m} / \mathrm{s}$. Only SS events were considered so as to focus on the magnitude scaling without possible complications due to mechanism-dependent scaling; almost $60 \%$ of the data in the NGA-West2 database are from SS events. The curves are from the Boore et al. (2014; hereafter BSSA14) GMPEs and from stochastic-method simulations, using the AS00 model parameters, with the original and revised $D_{\mathrm{P}}$. Also shown by a dashed line for $R_{\mathrm{JB}}=2 \mathrm{~km}$ is the result of not using the AS00 finite-fault adjustments to modify the distance used in the simulations (for $R_{\mathrm{JB}}=60 \mathrm{~km}$ the adjustments were so small that the simulations with and without the adjustments were almost the same). The color version of this figure is available only in the electronic edition.

show that the method predicts ground motions essentially as well as more complicated, physics-based simulation methods when judged in terms of the resulting response spectra (e.g., Hartzell et al., 1999). We feel that updating the duration model for active tectonic regions is warranted for the following reasons:

- The stochastic method remains a popular choice to simulate response spectra at all frequencies, especially in lowto-moderate seismicity regions (e.g., Douglas et al., 2013; Rietbrock et al., 2013).

- It is a key part of the hybrid-empirical method of developing ground-motion prediction equations (Pezeshk et al., 2011; Campbell, 2014).

- It remains a necessary high-frequency component of hybrid (physics and stochastic) simulations (e.g., Frankel, 2009; Graves and Pitarka, 2010).

- It is an essential part of stochastic finite-fault simulations (e.g., Motazedian and Atkinson, 2005; Boore, 2009; Ghofrani et al., 2013).
On the basis of data from many thousands of earthquakes accumulated as part of the PEER NGA-West2 project, we found that the path duration is longer than that given by the duration $0.05 R$ assumed in a number of studies. The revised duration is generally in relatively good agreement with that from several recent studies, at least for distances within about $50 \mathrm{~km}$. We emphasize that our revised path duration function is for use in the stochastic-method simulations and is not a general description of how duration depends on magnitude, distance, and frequency. Consistency is an important concern in using the revised duration. It is tied to a particular assumed shape of an exponential shaping window, and it must be used with that shape in future simulations.

\section{Data and Resources}

The flatfile containing ground-motion intensity measures and metadata for the PEER NGA-West2 project is available from $\mathrm{http}: / /$ peer.berkeley.edu/ngawest $2 /$ databases/ 
(last accessed February 2014). The time series and duration measures computed from the time series are not publicly available at this time, but the plan is to make them available within a year. The bulk of the data analysis was done using the program R, available from http://www.r-project.org/ (last accessed February 2014), and many of the figures were prepared using CoPlot (www.cohort.com; last accessed February 2014). All simulations were made using programs in the SMSIM package, the latest version of which can be obtained from the online software link on http://www.daveboore. com (last accessed July 2014); their use is described in Boore (2005).

Support for the statement that the high-frequency spectral level of the AS00 spectral model equals that for a singlecorner frequency model with a stress parameter of 88 bars is given in http://www.daveboore.com/daves_notes/What_SCF_ stress_param_is_consistent_with_the_AS00_source_model .pdf, last accessed July 2014.

Information about four recent building codes can be found in http://earthquake.usgs.gov/hazards/designmaps/ usdesigndoc.php; last accessed May 2014).

\section{Acknowledgments}

This paper was prepared as an account of work sponsored by an agency of the U.S. Government. Neither the U.S. Government nor any agency thereof, nor any of their employees, makes any warranty, expressed or implied, or assumes any legal liability or responsibility for any third party's use, or the results of such use, of any information, apparatus, product, or process disclosed in this report, or represents that its use by such third party would not infringe privately owned rights. The views expressed in this paper are not necessarily those of the U.S. Nuclear Regulatory Commission. We thank Annemarie Baltay for useful discussions, Tadahiro Kishida for providing some of the time series used in the article, and $\mathrm{K}$. Afshari and J. P. Stewart for providing us with their presentation given at the 2013 Southern California Earthquake Center (SCEC) annual meeting. We also thank Brad Aagaard, Annemarie Baltay, Giovanna Cultrera, Tom Hanks, and an anonymous reviewer for useful comments on the paper.

\section{References}

Ancheta, T. D., R. B. Darragh, J. P. Stewart, E. Seyhan, W. J. Silva, B. S. J. Chiou, K. E. Wooddell, R. W. Graves, A. R. Kottke, D. M. Boore, T. Kishida, and J. L. Donahue (2014). NGA-West2 database, Earthq. Spectra 30, doi: 10.1193/070913EQS197M.

Atkinson, G. M. (1993). Notes on ground motion parameters for eastern North America: Duration and H/V ratio, Bull. Seismol. Soc. Am. 83, 587-596.

Atkinson, G. M. (1995). Attenuation and source parameters of earthquakes in the Cascadia region, Bull. Seismol. Soc. Am. 85, 1327-1342

Atkinson, G. M., and D. M. Boore (1995). Ground motion relations for eastern North America, Bull. Seismol. Soc. Am. 85, 17-30.

Atkinson, G. M., and W. Silva (2000). Stochastic modeling of California ground motions, Bull. Seismol. Soc. Am. 90, 255-274.

Bommer, J. J., and A. Martínez-Pereira (1999). The effective duration of earthquake strong motion, J. Earthq. Eng. 3, 127-172.

Bommer, J. J., P. J. Stafford, and J. E. Alarcón (2009). Empirical equations for the prediction of the significant, bracketed, and uniform duration of earthquake ground motion, Bull. Seismol. Soc. Am. 99, 3217-3233

Boore, D. M. (1983). Stochastic simulation of high-frequency ground motions based on seismological models of the radiated spectra, Bull. Seismol. Soc. Am. 73, 1865-1894.
Boore, D. M. (2003). Prediction of ground motion using the stochastic method, Pure Appl. Geophys. 160, 635-676.

Boore, D. M. (2005). SMSIM-Fortran Programs for Simulating Ground Motions from Earthquakes: Version 2.3-A Revision of OFR 9680-A, U.S. Geol. Surv. Open-File Rept. 00-509, revised 15 August 2005, $55 \mathrm{pp}$.

Boore, D. M. (2009). Comparing stochastic point-source and finite-source ground-motion simulations: SMSIM and EXSIM, Bull. Seismol. Soc. Am. 99, 3202-3216.

Boore, D. M. (2014). What do data used to develop ground-motion prediction equations tell us about motions near faults? Pure Appl. Geophys. 171, doi: 10.1007/s00024-013-0748-9.

Boore, D. M., and E. M. Thompson (2012). Empirical improvements for estimating earthquake response spectra with random-vibration theory, Bull. Seismol. Soc. Am. 102, 761-772.

Boore, D. M., J. P. Stewart, E. Seyhan, and G. M. Atkinson (2014). NGA-West 2 equations for predicting PGA, PGV, and 5\%-Damped PSA for shallow crustal earthquakes, Earthq. Spectra 30, doi: 10.1193/070113EQS184M.

Bora, S. S., F. Scherbaum, N. Kuehn, and P. Stafford (2014). Fourier spectral- and duration models for the generation of response spectra adjustable to different source-,propagation-, and site conditions, Bull. Earthq. Eng. doi: 10.1007/s10518-013-9482-z.

Campbell, K. W. (2014). An evaluation of eastern North American groundmotion models developed using the hybrid empirical method, Bull. Seismol. Soc. Am. 104, 347-359.

Douglas, D., B. Edwards, V. Convertito, N. Sharma, A. Tramelli, D. Kraaijpoel, B. M. Cabrera, N. Maercklin, and C. Troise (2013). Predicting ground motion from induced earthquakes in geothermal areas, Bull. Seismol. Soc. Am. 103, 1875-1897.

Frankel, A. (2009). A constant stress-drop model for producing broadband synthetic seismograms: Comparison with the Next Generation Attenuation relations, Bull. Seismol. Soc. Am. 99, 664-680.

Ghofrani, H., and G. M. Atkinson (2014). Duration of the 2011 Tohoku earthquake ground motions, J. Seismol., doi: 10.1007/s10950-014-9447-y.

Ghofrani, H., G. M. Atkinson, K. Goda, and K. Assatourians (2013). Stochastic finite-fault simulations of the 2011 Tohoku, Japan, earthquake, Bull. Seismol. Soc. Am. 100, 2095-2123.

Graves, R. W., and A. Pitarka (2010). Broadband ground-motion simulation using a hybrid approach, Bull. Seismol. Soc. Am. 100, 2095-2123.

Hanks, T. C., and R. K. McGuire (1981). The character of high-frequency strong ground motion, Bull. Seismol. Soc. Am. 71, 2071-2095.

Hartzell, S., S. Harmsen, A. Frankel, and S. Larsen (1999). Calculation of broadband time histories of ground motion: Comparison of methods and validation using strong-ground motion from the 1994 Northridge earthquake, Bull. Seismol. Soc. Am. 89, 1484-1504.

Herrmann, R. B. (1985). An extension of random vibration theory estimates of strong ground motion to large distances, Bull. Seismol. Soc. Am. 75, $1447-1453$

Kempton, J. J., and J. P. Stewart (2006). Prediction equations for significant duration of earthquake ground motions considering site and nearsource effects, Earthq. Spectra 22, 985-1013.

Lee, J., and R. A. Green (2014). An empirical significant duration relationship for stable continental regions, Bull. Earthq. Eng. 12, 217-235.

Malagnini, L., K. Mayeda, R. Uhrhammer, A. Akinci, and R. B. Herrmann (2007). A regional ground-motion excitation/attenuation model for the San Francisco region, Bull. Seismol. Soc. Am. 97, 843-862.

Motazedian, D., and G. M. Atkinson (2005). Stochastic finite-fault modeling based on a dynamic corner frequency, Bull. Seismol. Soc. Am. 95, 9951010.

Ou, G.-B., and R. B. Herrmann (1990a). Estimation theory for strong ground motion, Seismol. Res. Lett. 61, 99-107.

Ou, G.-B., and R. B. Herrmann (1990b). A statistical model for ground motion produced by earthquakes at local and regional distances, Bull. Seismol. Soc. Am. 80, 1397-1417.

Pezeshk, S., A. Zandieh, and B. Tavakoli (2011). Hybrid empirical groundmotion prediction equations for eastern North America using NGA 
models and updated seismological parameters, Bull. Seismol. Soc. Am. 101, 1859-1870.

Pitarka, A., L. A. Dalguer, S. M. Day, P. G. Somerville, and K. Dan (2009) Numerical study of ground-motion differences between buriedrupturing and surface-rupturing earthquakes, Bull. Seismol. Soc. Am. 99, 1521-1537.

Raoof, M., R. B. Herrmann, and L. Malagnini (1999). Attenuation and excitation of three-component ground motion in Southern California, Bull. Seismol. Soc. Am. 89, 888-902.

Rietbrock, A., F. Strasser, and B. Edwards (2013). A stochastic earthquake ground-motion prediction model for the United Kingdom, Bull. Seismol. Soc. Am. 103, 57-77.

Saragoni, G. R., and G. C. Hart (1974). Simulation of artificial earthquakes, Earthq. Eng. Struct. Dynam. 2, 249-267.

Trifunac, M. D., and A. G. Brady (1975). A study on the duration of strong earthquake ground motion, Bull. Seismol. Soc. Am. 65, $581-626$.

Vanmarcke, E. H., and S.-S. P. Lai (1980). Strong-motion duration and rms amplitude of earthquake records, Bull. Seismol. Soc. Am. 70, 1293-1307.
U.S. Geological Survey

MS 977

345 Middlefield Road

Menlo Park, California 94025

boore@usgs.gov

(D.M.B.)

Department of Geological Sciences

San Diego State University

San Diego, California 92182

ethompson@mail.sdsu.edu

(E.M.T.)
Manuscript received 1 March 2014;

Published Online 12 August 2014 